\title{
Seroprevalencia de Mycobacterium avium Subsp. paratuberculosis (MAP) en una granja de ganado de carne de bosque húmedo tropical en Caucasia, Antioquia, Colombia
}

\author{
Seroprevalence of Mycobacterium avium Subsp. paratuberculosis (MAP) in a \\ beef cattle farm of a tropical moist forest in Caucasia, Antioquia, Colombia
}

\author{
VÉLEZ A. MARIA ${ }^{1}$ MV, RENDÓN D. YINEDY ${ }^{1}$ MV, VALENCIA R. ANDRÉS ${ }^{1}$ MV, \\ RAMÍREZ V. NICOLÁS ${ }^{1}$ Ph.D, FERNÁNDEZ-S JORGE ${ }^{1 *}$. Ph.D.
}

${ }^{1}$ Universidad de Antioquia, Escuela de Medicina Veterinaria, Facultad de Ciencias Agrarias, Epidemiología y Salud Pública Veterinaria, Centauro, Calle 70 No. 52-21, Medellín, Colombia.

Keywords:

Diagnosis, Johne's disease, beef cattle, paratuberculosis, tropical moist forest

Palabras Clave:

Diagnóstico, enfermedad de Johne, ganado de carne, paratuberculosis, bosque húmedo tropical

INFORMACIÓN

Recibido: 14-02-2016;

Aceptado: 20-05-2016.

Correspondencia autor:

jorge.fernandez@udea.edu.co

\section{Abstract}

The present study determines the seroprevalence of MAP in beef cattle of a farm in tropical moist forest located in the municipality of Caucasia. Blood samples from all animals over two years of age $(n=151)$ were obtained together with information on the epidemiological characteristics of the animal (age, breed, sex, parity, reproductive state, days postpartum). Serum samples were analyzed with an Enzyme-Linked Immunosorbent Assay (ELISA) and the information analyzed using descriptive and analytic statistics (two-way frequency tables). ELISA results showed a seroprevalence of $33.8 \%$ (IC 95\% 26.1-41.4) showed statistically significant association between ELISA results and breed. According to the results, high seroprevalence of paratuberculosis level was determined in a beef farm for the first time in Colombia. Further studies on beef cattle herds should be carried out to increase the knowledge of the prevalence of this disease in the country.

\section{Resumen}

El presente estudio se realizó para determinar la seroprevalencia a MAP en ganado de carne de una granja en bosque húmedo tropical ubicada en el municipio de Caucasia. Se tomaron muestras de sangre de todos los bovinos mayores de dos años $(n=151)$ y se obtuvo información de características individuales (edad, raza, sexo, número de partos, estado reproductivo y días posparto). Las muestras de suero fueron analizadas mediante el Ensayo por inmunoabsorción ligado a enzimas (ELISA), mientras que la información fue analizada usando estadística descriptiva y analítica (Prueba de chi cuadrado y prueba exacta de Fisher). Los resultados de ELISA mostraron una seroprevalencia del 33.8\% (IC 95\% 26.1-41.4) y el análisis estadístico mostró asociación estadísticamente significativa entre el resultado de ELISA y la raza. De acuerdo a los resultados, un alto nivel de seroprevalencia fue determinado en ganado de carne por primera vez en Colombia. Futuros estudios en este tipo de ganado deben ser realizados para aumentar el conocimiento de la prevalencia de la enfermedad en el país. 


\section{Introducción}

Mycobacterium avium subespecie paratuberculosis (MAP) es el agente etiológico de la paratuberculosis (PTB) o enfermedad de Johne en rumiantes. La PTB es una enfermedad crónica que causa enteritis granulomatosa con linfoadenitis y linfangitis de linfonodos mesentéricos de estos animales. La PTB tiene una distribución mundial y un impacto económico significativo (CLARKE, 1997). Sin embargo, debido a que la mayoría de los casos de la enfermedad de Johne son subclínicos y datos sobre prevalencia son escasos, es difícil estimar las consecuencias económicas de esta enfermedad para individuos, hatos o el país. La enfermedad de Johne tiene implicaciones serias para el comercio nacional e internacional de animales vivos y germoplasma, debido a que la enfermedad e Johne se propaga con mayor efectividad a través del movimiento de animales infectados que contaminan un nuevo ambiente estableciendo el escenario para la masiva exposición de más animales, y a que MAP ha sido aislado de semen y embriones de reproductores afectados, aunque se desconoce la efectividad de esta ruta de transmisión. El comercio internacional de animales ha contribuido a la diseminación global de la enfermedad de Johne y la Organización Mundial de Sanidad Animal (OIE) considera la enfermedad de Johne o paratuberculosis como una enfermedad de gran importancia global (COMMITTEE ON DIAGNOSIS AND CONTROL OF JOHNE'S DISEASE, 2003). En Colombia el Instituto Colombiano Agropecuario (ICA) considera a la paratuberculosis como una enfermedad común a varias especies de denuncia obligatoria (ICA, 2015).

En ganado de carne el impacto económico no ha sido estimado con precisión. La baja prevalencia intrahato aunado a la escasez de registros completos en muchas granjas de carne hacen que la determinación del impacto económico en ganado de carne sea difícil. La PTB puede causar pérdidas por muerte, venta de animales con pesos por debajo de lo esperado y costos de remplazo asociados a la enfermedad. En ganado de carne se asume una reducción de la producción de leche como ocurre en ganado lechero, lo que conlleva a que los pesos ajustados al destete de los terneros de vacas infectadas con MAP sean menores (ROUSSEL, 2011). Las pérdidas económicas asociadas en vacas de ganado de carne seropositivas a MAP o de las cuales se aisló MAP de materia fecal fueron estimadas en US\$ 57.49/ternero, para vacas con resultados de ELISA fuertemente positivos; y US\$156.60/ternero y US\$109.23/ternero para vacas clasificadas como altamente excretoras y moderadamente excretoras de MAP, respectivamente (BHATTARAl et al. 2013a). Otros costos menos obvios podrían incluir la pérdida potencial en ventas en el caso de productores de ganado puro, el costo de pérdida de material genético valioso, pérdida de mercados de exportación y pérdida de la confianza del consumidor. Muchos de estos costos son ocultos o costos de oportunidad (ROUSSEL, 2011). Un estudio previo estimó que la pérdida anual por cada animal infectado, basada en información suministrada por productores, fue de US\$225 (95\% CR: \$89-\$457). La misma estimación utilizando información suministrada por médicos veterinarios fue de $\$ 250$ (\$82-\$486). La pérdida anual estimada debida la enfermedad de Johne en una granja de 100 vacas con una prevalencia verdadera de $7 \%$ fue de US $\$ 1644$ (\$625-\$3250) basada en información suministrada por los productores. De igual manera, la pérdida anual estimada basada en información obtenida de médicos veterinarios fue de US\$1747 (\$575-\$3375, BHATTARAl et al. 2013b)

En los últimos años se ha planteado la pregunta sobre si MAP (el agente etiológico de la enfermedad de Johne) podría causar la enfermedad de Crohn humana o si es un hallazgo incidental sin importancia clínica en esta última enfermedad. El origen de esta asociación entre la enfermedad de Johne y la enfermedad de Crohn ha sido la similitud clínica y patológica entre las dos enfermedades (ambas granulomatosas del intestino con predilección por el íleon). Según otra perspectiva, la similitud ha sido exagerada, pues al ser comparadas también pueden verse varias diferencias clínicas significativas en el estado preclínico y clínico, los síntomas y signos gastrointestinales, las manifestaciones gastrointestinales, el curso clínico, y diferencias patológicas en la localización de la lesión, las características macroscópicas y la apariencia microscópica. Las similitudes y las diferencias han sido interpretadas por expertos a favor y en oposición a la visión de MAP como causa de la enfermedad de Crohn (COMMITTEE ON DIAGNOSIS AND CONTROL OF JOHNE'S DISEASE, 2003). 
En el mundo, la paratuberculosis en ganado de carne es menos prevalente que en ganado de leche (ROUSSEL, 2011). Diversos estudios muestran una mayor presentación en ganado de leche y doble propósito frente al ganado de carne, no sólo a nivel animal, sino también por hatos (MERKEL et al., 1987; ROUSSEL, 2011). En América Latina y el Caribe se estima una prevalencia (mayoritariamente en ganado de leche) de $16,9 \%$ (95 \% Cl 13,2-20,5) y $75,8 \%$ $(95 \% \mathrm{Cl} 50,1-101,5)$ a nivel individual y a nivel de hato, respectivamente (FERNÁNDEZ-S et al., 2014). En Colombia, se han hecho estudios sobre MAP y PTB, todos en ganado de leche (ZAPATA et al., 2010; RAMÍREZ et al. 2001; RAMÍREZ et al., 2011; FERNÁNDEZ-S et al., 2011a; FERNÁNDEZ-S et al., 2011b; RAMÍREZ-G y MALDONADO-E, 2013) pero no se cuenta con una prevalencia nacional o prevalencias regionales y no se han realizado estudios de la enfermedad en ganado de carne (CORREA-V et al., 2015). En el caso colombiano, un estudio realizado en el trópico bajo en el departamento de Córdoba obtuvo una seroprevalencia del 25\% en un hato de ganado lechero (DE WAARD, 2010). Es importante establecer la magnitud de la presentación de la PTB en ganado de carne para proponer medidas de control de la enfermedad adecuadas y así evitar pérdidas en la producción, la transmisión y propagación entre los hatos.

El objetivo de este estudio es determinar la seroprevalencia de MAP en una granja de ganado de carne de bosque húmedo tropical en Caucasia, Antioquia, Colombia y explorar la asociación entre factores individuales de los animales con el resultado de ELISA para MAP.

\section{Materiales y métodos}

Tipo de estudio: Se realizó un estudio observacional, analítico seccional cruzado (DOHOO et al., 2010), el cual fue avalado por el Comité de Ética para la Experimentación con Animales (CEEA) de la Universidad de Antioquia, según acta 87 del 30 de enero de 2014.

Granja: La granja está ubicada en el municipio de Caucasia, región del Bajo Cauca, departamento de Antioquia (Colombia), en zona de vida Bosque
Húmedo Tropical, a 50 metros sobre el nivel del mar (msnm), con precipitación anual de 2.100 milímetros (mm), temperatura promedio de $26^{\circ} \mathrm{C}$, y humedad relativa del $75 \%$. La granja cuenta con una extensión total de 264.48 hectáreas y un total de 266 bovinos repartidos en diferentes lotes, según su edad y estado reproductivo. La granja está certificada como hato libre de brucelosis y tuberculosis bovina (pendiente de recertificación en ésta última). Actualmente es un hato cerrado, pues tienen sistema de autoreemplazo. Sin embargo, los animales eran traídos anteriormente de una granja del mismo propietario ubicada en el municipio de Gómez Plata, región del Nordeste, departamento de Antioquia (Colombia), en zona de vida Bosque Muy Húmedo Premontano, a $1.080 \mathrm{msnm}$, con precipitación anual de $1.800 \mathrm{~mm}$, temperatura promedio de $25^{\circ} \mathrm{C}$, y humedad relativa de $80 \%$, la cual, a su vez, recibía animales de una granja del mismo propietario ubicada en el municipio de San Pedro de Los Milagros, región Norte, departamento de Antioquia (Colombia), en zona de vida Bosque Húmedo Montano Bajo, a 2.400 msnm, con precipitación anual de $1575 \mathrm{~mm}$, temperatura promedio de $15^{\circ} \mathrm{C}$, y humedad relativa del $72 \%$. Esta última granja ha presentado casos clínicos y diagnóstico positivo a MAP según diferentes estudios previos (ZAPATA et al., 2010; RAMÍREZ et al., 2011; RAMÍREZ-G y MALDONADO-E, 2013).

Animales: A todos los bovinos mayores de dos años, de ambos sexos, de todas las razas, cruces, y estados reproductivos de la granja $(n=151)$ se les tomó muestra de sangre en agosto de 2014. Todos los animales fueron reportados como asintomáticos para PTB y no habían sido muestreados antes para esta enfermedad.

Muestras: Las muestras de sangre de los animales se tomaron de la vena coccígea previa desinfección del área con alcohol antiséptico. Después de permitir la retracción del coágulo sanguíneo a temperatura ambiente, el suero se separó mediante centrifugación a 2000 rpm durante cinco minutos. Las muestras de suero fueron refrigeradas a $4^{\circ} \mathrm{C}$ hasta su llegada al Laboratorio Clínico y de Serología de la Unidad de Diagnóstico de la Facultad de Ciencias Agrarias en la Universidad de Antioquia, donde fueron congeladas a $-20^{\circ} \mathrm{C}$ hasta su análisis posterior por medio de la prueba de ELISA. 
ELISA: La prueba de ELISA se realizó empleando el kit comercial preabsorbido ID VET, ID Screen ${ }^{\circledR}$ Paratuberculosis Indirect, Screening test (Grabels, Francia) según el protocolo del fabricante. Para establecer el resultado de ELISA se determinó la Densidad Óptica Media del Control Positivo (DOCP) y del Control Negativo (DOCN). Posteriormente se obtuvo la proporción entre las Densidades Ópticas Medias de los controles positivos y negativos (DOCP/DOCN), procedimiento por el cual se confirmó la validez del ensayo. Cada muestra fue analizada una sola vez para obtener el porcentaje $\mathrm{S} / \mathrm{P}(\mathrm{S} / \mathrm{P} \%)$ mediante la fórmula $\mathrm{S} / \mathrm{P} \%=$ DO muestra - DOCN x 100 /_DOCP - DOCN. Los resultados de ELISA que el kit diagnóstico clasificó como dudosos fueron considerados como positivos para los análisis posteriores.

Información sobre características individuales de los animales: Simultáneamente a la toma de muestras de sangre de los animales se recolectó información acerca de edad, raza, sexo, número de partos, estado reproductivo, y días posparto de cada animal muestreado, con el fin de explorar la asociación de factores individuales de los animales con estatus serológico de MAP. Para esto se empleó un formato el cual fue completado por el administrador de la granja.

Análisis de datos: Los datos se almacenaron en hojas de cálculo Excel (Microsoft Corp., Redmond, WA) y luego se exportaron al software estadístico Stata 12.0 (StataCorp, 2011) para su análisis. Se efectuó estadística descriptiva para todas las variables de interés. Con la información de las variables y el resultado del ELISA se efectuó análisis de la asociación de los factores individuales del animal (edad, raza, sexo, número de partos, estado reproductivo, y días posparto) con la seropositividad a MAP, esto último por medio de la prueba de Chi cuadrado de Pearson y para aquellas variables que presentaban menos de cinco datos en la tabla de contingencia se utilizó la prueba exacta de Fisher. Se estableció un nivel de significancia de $p<0,05$.

\section{Resultados}

Características individuales de los animales muestreados: La media de la edad en años, del número de partos, y del número de días posparto en los animales muestreados fue de 6.1 años, 3 partos y 290.7 días posparto, respectivamente (Tabla 1). Se obtuvo información sobre la raza en 145 de los 151 animales muestreados, estableciéndose que los animales pertenecían a las razas Blanco Orejinegro (BON, 1,4\%, 2/143), Brahman (46,9\%, 67/143) y cruce BON por Brahman $(51,7 \%, 74 / 143)$. Esta variable se re-categorizó para el análisis estadístico en Brahman $(46,9 \%, 67 / 143)$ y Otra (BON o Cruce BON x Brahman, 53,1\%, 76/143, Tabla 2). De los 151 animales muestreados, 148 eran hembras y tres eran machos. De 144 animales con datos reproductivos disponibles, se estableció que los tres machos son reproductores actualmente en uso y que las hembras se encontraban preñadas $(n=62)$, recién paridas $(n=44)$, o vacías $(n=35)$ al momento del muestreo.

ELISA: La prueba de ELISA en suero sanguíneo arrojó que el 33,8\% (51/151, IC 95\% 26,1-41,4) de los animales fueron positivos, mientras el $66,2 \%$ (IC $95 \% 0,58-0,73)$ fueron negativos para la presencia de anticuerpos contra MAP. El 3,3\% (5/151) de los animales arrojó resultados clasificados por la prueba de ELISA como dudosos, los cuales se consideraron como positivos y están incluidos dentro estos últimos. En consecuencia, la prevalencia aparente obtenida en este estudio fue de $33.8 \%$ (51/151).

Tabla 1. Variables individuales cuantitativas de bovinos adultos muestreados para Mycobacterium avium subsp. paratuberculosis (MAP) en una granja de ganado de carne de bosque húmedo tropical en Caucasia, Antioquia, Colombia

\begin{tabular}{|c|c|c|c|c|c|}
\hline Variable & $\begin{array}{c}\text { Número } \\
\text { observaciones }\end{array}$ & Media & Desviación estándar & Min. & Max. \\
\hline Edad (años) & 145 & 6,1 & $\pm 2,9$ & 2 & 14,34 \\
\hline Número de partos & 135 & 3 & $\pm 2,3$ & 0 & 10 \\
\hline Días postparto & 125 & 290,7 & $\pm 210,3$ & 2 & 848 \\
\hline
\end{tabular}


Factores asociados al resultado de ELISA: El análisis de factores individuales mostró asociación estadísticamente significativa entre el resultado de ELISA y la raza $(p=0,033)$ en la prueba exacta de Fisher. En el presente estudio la seropositividad a MAP fue proporcionalmente más alta en los animales con mayor presencia genética Brahman: $0 \%$ en el BON puro, $25.7 \%$ en el cruce BON x Brahman, y $41.8 \%$ en el Brahman puro (datos no mostrados). Para el resto de los factores individuales (edad, raza, sexo, número de partos, estado reproductivo, y días posparto) no se observó asociación estadísticamente significativa ( $p>0,05$, Tabla 2).

\section{Discusión}

En el presente estudio se determinó la seroprevalencia de MAP y se exploró la asociación entre factores del individuo y el resultado del ELISA en una granja de ganado bovino destinado a la producción de carne, ubicado en zona de vida Bosque Húmedo Tropical del municipio de Caucasia, región del Bajo Cauca en Antioquia, Colombia.

El resultado de seroprevalencia obtenido en el presente estudio $(33,8 \%)$ es similar al obtenido en estudios previos en ganado de carne (cruces Bos taurus (BT) con Bos indicus (BI)) usando una prueba de ELISA no preabsorbida en un matadero en el estado de Pará, Brasil 35,4\% (SILVA, 2005). Por otro lado, el resultado es mayor al resultado de prevalencia a nivel animal obtenido en ganado de carne en Argentina 13,8\% (MOREIRA et al., 1994), Estados Unidos 4,4\%, 3,9\%, 8,0\%, y 3,0\%) (TURNQUIST et al., 1991; PENCE et al., 2003; HILL et al., 2003; ROUSSEL et al., 2005), Canadá (0,8 y 1,5\% (WALDNER et al., 2002; SCOTT et al., 2007), y China 11,17\% (SUN et al., 2007). Sin embargo, al comparar los resultados del presente estudio con aquellos estudios que reportan un resultado de seroprevalencia intrahato (obtenidos por medio de la prueba de ELISA) el resultado de prevalencia del presente estudio es superior

Tabla 2. Variables individuales según status serológico a Mycobacterium avium subsp. paratuberculosis de bovinos adultos en una granja de ganado de carne de bosque húmedo tropical en Caucasia, Antioquia, Colombia

\begin{tabular}{|c|c|c|c|c|c|}
\hline \multirow{2}{*}{ Variable } & \multirow{2}{*}{ Categoría } & \multirow{2}{*}{$\begin{array}{c}\text { Número de } \\
\text { observaciones }\end{array}$} & \multicolumn{2}{|c|}{ Resultado ELISA } & \multirow{2}{*}{$p$} \\
\hline & & & Positivo & Negativo & \\
\hline \multirow[t]{3}{*}{ Edad } & $\leq 5$ años & 72 & 23 & 49 & $0,905^{\mathrm{a}}$ \\
\hline & $>5$ años & 73 & 24 & 49 & \\
\hline & Total & 145 & 47 & 98 & \\
\hline \multirow[t]{3}{*}{ Raza } & Brahman & 67 & 28 & 39 & $0,033^{a}$ \\
\hline & $\begin{array}{l}\text { Otra (BON o Cruce } \\
\text { BON x Brahman) }\end{array}$ & 76 & 19 & 57 & \\
\hline & Total & 143 & 47 & 96 & \\
\hline \multirow[t]{3}{*}{ Sexo } & Macho & 3 & 1 & 2 & $0,987^{b}$ \\
\hline & Hembra & 148 & 50 & 98 & \\
\hline & Total & 151 & 51 & 100 & \\
\hline \multirow[t]{3}{*}{ Número de partos } & $\leq 2$ & 65 & 22 & 43 & $0,903^{a}$ \\
\hline & $>2$ & 70 & 23 & 47 & \\
\hline & Total & 135 & 45 & 90 & \\
\hline \multirow[t]{3}{*}{ Días posparto } & $0-100$ & 24 & 10 & 14 & $0,352^{\mathrm{a}}$ \\
\hline & $>100$ & 101 & 32 & 69 & \\
\hline & Total & 125 & 42 & 83 & \\
\hline
\end{tabular}


al de WALDNER et al., 2002 (prevalencias intrahato entre 2 y 12,8\%) y ROUSSEL et al., 2005 (prevalencias intrahato entre $2 y>12 \%$ ), pero similares a los del estudio de ROUSSEL et al., 2007 cuyas prevalencias intrahato en la mayoría de las granjas analizadas estuvo entre 23 y $75 \%$.

Aunque el resultado de seroprevalencia encontrado resultó mayor a lo esperado y lo reportado en bovinos para Latinoamérica, el Caribe, y Colombia (FERNÁNDEZ et al., 2014; CORREA et al., 2015), el resultado podría explicarse por la entrada de animales desde una de las granjas del propietario, en la cual ya se ha demostrado la presencia de MAP (ZAPATA et al., 2010; RAMÍREZ et al., 2011, RAMÍREZ-G y MALDONADO, 2013); y que es considerada como una de las principales formas de transmisión de la enfermedad de granjas de carne infectadas a granjas libres (ROUSSEL, 2011).

Adicionalmente, existe evidencia de la reacción cruzada con micobacterias ambientales en algunos hatos de ganado $\mathrm{BI}$, lo que ha significado mayores seroprevalencias y que han llevado a sugerir la posible explicación de una mayor seroreactividad del ganado $\mathrm{BI}$ a los antígenos micobacteriales, ya sean por MAP o por micobacterias ambientales (ROUSSEL et al., 2005; ROUSSEL, 2011; NORBY et al 2007). En estos trabajos también se han empleado ELISAs preabsorbidos (HerdChek, IDEXX Laboratories Inc, Westbrook, Me. y ParaCheck, CSL/Biocor, Omaha, Neb.) reportando el mismo resultado hasta el punto de sugerir la confirmación de los datos de alta seropositividad con otro ELISA, o una prueba directa (ROUSSEL et al. 2007). En el presente estudio, dicha confirmación estaba más allá del objetivo de nuestro estudio, pero abre perspectivas para investigaciones futuras en la misma granja. Aunque la especificidad del ELISA utilizado en el presente estudio se reporta por encima de 99\% (FRY et al. 2008; KÖHLER et al. 2008), la posibilidad de falsos positivos, aunque muy baja, existe.

La prevalencia estimada en el presente estudios corresponde a la prevalencia aparente, la cual una vez ajustada con datos de sensibilidad y especificidad del ELISA utilizado, muy probablemente revelaran que la prevalencia intrahato ajustada (prevalencia verdadera) es superior a la estimada. Dicho ajuste no se presenta debido a que los datos de sensibilidad y especificidad del ELISA reportados (FRY et al. 2008; KÖHLER et al. 2008) provienen de evaluaciones realizadas en otros países, en ganado lechero y bajo condiciones productivas y estacionales muy diferentes. Según lo sugerido por NIELSEN Y TOFT, 2008 y NIELSEN Y TOFT, 2009 , previo a un estudio de prevalencia, es ideal que la exactitud del test diagnóstico a utilizar en el estudio fuese evaluada en la población objetivo. A su vez, para esta evaluación de exactitud, la sensibilidad y especificidad son necesarias y deberían haber sido estimadas previamente en una población geográficamente comparable y empleando pruebas diagnósticas (de referencia) como cultivo (o PCR fecal) que por ahora son difíciles de realizar por las dificultades en el transporte y conservación de este tipo de muestras desde el sitio donde está ubicada la granja. En caso de que esta estimación previa de la sensibilidad y especificidad "local" no se haga o no se haga apropiadamente, se pueden obtener valores de prevalencia inexactos e improbables (como por encima del 100\%, NIELSEN Y TOFT, 2009). Este tipo de evaluación de exactitud de la prueba diagnóstica no era realizable en nuestras condiciones debido a las restricciones presupuestales y se salía del propósito del presente estudio y se prefirió reportar la prevalencia aparente en vez de la prevalencia verdadera, hasta que no contemos con estudios de evaluación y validación proveniente de contextos productivos locales.

La asociación estadísticamente significativa entre el resultado de ELISA y la raza $(p=0.033)$ concuerda con la aparente diferencia de susceptibilidad entre razas de carne, al menos cuando se estima prevalencia promedio de pruebas serológicas, como lo demuestra un estudio en el que se encontró mayor prevalencia en Brahman comparado con Angus en un hato de tamaño moderado, en el cual además se encontró que entre el ganado cruzado del hato, la seroprevalencia se correlacionó positivamente con el porcentaje de Brahman en el pedigrí (ELZO et al., 2006; ROUSSEL, 2011). De la misma manera, se encontró mayor seroprevalencia en hatos de ganado puro de carne $\mathrm{Bl}$ y en hatos con influencia BI que en hatos BT (ROUSSEL et al., 2005; ROUSSEL, 2011). En el ámbito nacional, 
un estudio reciente en un hato de ganado BON y sus cruces en Antioquia, en el cual se determinó una seroprevalencia de 4\% (2/50; TUBERQUIA et al. 2015) no reveló ninguna asociación estadísticamente significativa entre resultado del ELISA y la raza de los animales.

Las diferencias de raza juegan un papel fundamental en la genética de la resistencia a las enfermedades y pueden ser usadas como una herramienta para el control de la enfermedad (VIR SINGH et al. 2013; VAN HULZEN et al. 2011). Los efectos y las diferencias de la raza con respecto a la infección por MAP han sido estudiados y descritas en diferentes poblaciones bovinas (CLARKE, 1997).

De acuerdo con ROUSSEL (2011) la mayor diferencia entre ganado de carne y ganado de leche cuando se considera la trasmisión y el control de paratuberculosis involucra las prácticas de manejo de los terneros. El ternero de leche es separado de la madre dentro de las primeras 24 horas de nacido, mientras que los terneros de carne permanecen con las madres de 6 a 7 meses. Consecuentemente, la exposición de los terneros de carne al estiércol de animales adultos es mucho mayor en las explotaciones de carne que en las explotaciones de leche.
El presente estudio tuvo limitaciones asociadas con las dificultades para confirmar el diagnóstico serológico por medio de una prueba directa (cultivo, PCR) debido a las limitaciones logísticas para la conservación y transporte de muestras fecales desde la granja al laboratorio y con la ausencia de estimaciones de sensibilidad y especificidad en poblaciones comparables para poder estimar adecuadamente la prevalencia verdadera.

\section{Conclusión}

Los resultados de seroprevalencia $(33,8 \%)$ y el factor asociado al resultado de ELISA (raza, $p=0,033$ ) coinciden con reportes de estudios comparables y con lo conocido de la enfermedad en ganado de carne. Igualmente, los resultados revelan la presencia de la enfermedad en este tipo de producción en el país, por lo que se requieren estudios adicionales para determinar la magnitud y distribución de la enfermedad en las granjas de ganado de carne del país.

Agradecimientos: Departamento de Formación Académica de Haciendas y Unidad de Diagnóstico de la Facultad de Ciencias Agrarias, UdeA; Semillero de investigación Colciencias (Convocatoria 617 de 2013); Estrategia de Sostenibilidad CODI 2013-2014 de la UdeA.

\section{Referencias}

BHATTARAI, B.; FOSGATE, G.T.; OSTERSTOCK, J.B.; FOSSLER, C.P.; PARK, S.C.; ROUSSEL, A.J. 2013a Comparison of calf weaning weight and associated economic variables between beef cows with and without serum antibodies against or isolation from feces of Mycobacterium avium subsp paratuberculosis. J Am Vet Med Assoc. 243 (11):1609-15.

BHATTARAI, B.; FOSGATE G.T.; OSTERSTOCK, J.B:; FOSSLER, C.P.; PARK, S.C.; ROUSSEL, A.J. $2013 \mathrm{~b}$ Perceptions of veterinarians in bovine practice and producers with beef cow-calf operations enrolled in the US Voluntary Bovine Johne's Disease Control Program concerning economic losses associated with Johne's disease. Prev Vet Med. 112 (3-4):330-7.

CLARKE, C.J. 1997. The pathology and pathogenesis of paratuberculosis in ruminants and others species. J. Comp. Path. 116:217-261.

COMMITTEE ON DIAGNOSIS AND CONTROL OF JOHNE'S DISEASE. 2003. National Research Council of the National Academy of Sciences. Diagnosis and Control of Johne's Disease. Washington (DC). National Academies Press (US). 
CORREA-VALENCIA, N.; RAMÍREZ, N.; OLIVERA, M.; FERNÁNDEZ-SILVA, J. Sometido. Milk yield and lactation stage are associated with positive results to ELISA for Mycobacterium avium subsp. paratuberculosis in dairy cows from Northern Antioquia, Colombia (sometido 17.11.2015)

DE WAARD, J. 2010. ¿Ordeñando micobacterias del ganado? Impacto económico y en salud de Tuberculosis bovina y Paratuberculosis en Colombia. Rev.MVZ Córdoba 15 (2):2037-2040.

DOHOO, I.; MARTIN W.; STRYHN, H. 2010. Introduction to observational studies. Págs. 151-166. En: DOHOO, I.; MARTIN W.; STRYHN, H. Veterinary Research Epidemiology. 2nd edition. VER Inc. Charlottetown, PE Canada.

ELZO, M.A.; RAE, D.O.; LANHART, S.E.; WASDIN, J.G.; DIXON, W.P.; JONES. J.L. 2006. Factors associated with ELISA scores for paratuberculosis in an Angus-Brahman multibreed herd of beef cattle. J. Anim. Sci. 84:41-48.

FERNÁNDEZ-SILVA, J.; ABDULMAWJOOD, A.; AKINEDEN, O.; BÜLTE, M. 2011. Serological and molecular detection of Mycobacterium avium subsp. paratuberculosis in cattle of dairy herds in Colombia. Trop Anim Health Prod. DOI 10.1007/s11250-011-9833-1.

FERNÁNDEZ-SILVA, J.; ABDULMAWJOOD, A.; BULTE, M. 2011. Diagnosis and Molecular Characterization of Mycobacterium avium subsp. paratuberculosis from Dairy Cows in Colombia. Veterinary Medicine International, Volume 2011, Article ID 352561, doi:10.4061/2011/352561.

FERNÁNDEZ-SILVA, J.; CORREA-VALENCIA, N.; RAMÍREZ-VÁSQUEZ, N. 2014. Systematic review of the prevalence of paratuberculosis in cattle, sheep, and goats in Latin America and the Caribbean. Trop Anim Health Prod 46 (8):1321-1340.

FRY, M.P.; KRUZE, J.; COLLINS, M.T. 2008. Evaluation of four commercial enzyme-linked immunosorbent assays for the diagnosis of bovine paratuberculosis in Chilean dairy herds. J Vet Diagn Invest. (3):329-32.

HILL, B.; WEST, M.; BROCK, K. 2003. An estimated prevalence of Johne's disease in a subpopulation of Alabama beef cattle. J Vet Diagn Invest. (1):21-5.

INSTITUTO COLOMBIANO AGROPECUARIO (ICA). Resolución 3714 (octubre 20) de 2015, por la cual se establecen las enfermedades de declaración obligatoria en Colombia. Colombia.

KÖHLER, H.; BURKERT, B.; PAVLIK, I.; DILLER, R.; GEUE, L.; CONRATHS, F.J.; MARTIN, G. 2008. Evaluation of five ELISA test kits for the measurement of antibodies against Mycobacterium avium subspecies paratuberculosis in bovine serum. Berl Munch Tierarztl Wochenschr121 (5-6):203-10.

MANNING, E.; COLLINS, M. 2001. Mycobacterium avium subsp paratuberculosis: pathogen, pathogenesis and diagnosis. Review of Scientific Technology 20:133-150.

MERKEL, R.; WHIPPLE, D.; SACKS, J.; SNYDER, G. 1987.Prevalence of Mycobacterium paratuberculosis in ileocecal lymph nodes of cattle culled in United States. J Am Vet Med Assoc. 190 (6):676-80.

MOREIRA, A.R.; SPATH, E.J.; MORSELLA, C. 1994. Seroprevalence of Johne's disease in eleven districts of Buenos Aires, Argentina. Abstracts from Oral and Poster presentations at the Fourth International Colloquium on Paratuberculosis, Cambridge, United Kingdom, July 17-21, 1994, International Association for Paratuberculosis, Inc.p. 10. 
NIELSEN, S.S.; TOFT, N. 2008. Ante-mortem diagnosis of paratuberculosis: a review of accuracies of ELISA, interferon-gamma assay and faecal culture techniques, Vet Microbiol 129:217-235.

NIELSEN, S.S.; TOFT, N. 2009. A review of prevalences of paratuberculosis in farmed animals in Europe. Prev Vet Med. 88:1-14.

NORBY, B.; FOSGATE, G.T.; MANNING, E.J.; COLLINS, M.T.; ROUSSEL, A.J. 2007. Environmental mycobacteria in soil and water on beef ranches: association between presence of cultivable mycobacteria and soil and water physicochemical characteristics. Vet Microbiol. 124 (1-2):153-9.)

PAOLICCHI, F.; ZUMARRAGA, M.; GIOFFRE, A.; ZAMORANO, P.; MORSELLA, C.; VERNA, A.; CATALDI, A.; ALITO A.; ROMANO, M. 2003. Application of different methods for the diagnosis of Mycobacterium avium subsp paratuberculosis in a dairy cattle herd in Argentine. Journal of Veterinary Medicine Series "B" 50:20-26.

PENCE, M.; BALDWIN, C.; BLACK, C.J. 2003. The seroprevalence of Johne's disease in Georgia beef and dairy cull cattle. Vet Diagn Invest. 15:475-477.

RAMÍREZ, N.; GAVIRIA, G.; RESTREPO, L. 2001 (no publicado). Diagnóstico epidemiológico referente a varias patologías de bovinos en tres haciendas de la Universidad de Antioquia. Facultad de Ciencias Agrarias, Universidad de Antioquia.

RAMÍREZ-GARCÍA, R.; MALDONADO-ESTRADA, J. 2013. Detection of macrophages infected with Mycobacterium avium subspecies paratuberculosis in a cow with clinical stage IV of Johne's disease. A case report. Rev Colomb Cienc Pecu. 26:219-225.

RAMÍREZ, N.; RODRÍGUEZ, B.; FERNÁNDEZ J. 2011. Diagnóstico clínico e histopatológico de paratuberculosis bovina en un hato lechero en Colombia. Rev. MVZ Córdoba 16 (3):2742-2753.

ROUSSEL, A.J. 2011. Control of paratuberculosis in Beef Cattle. Vet Clin Food Anim. 27:593-598

ROUSSEL, A.; FOSGATE, G.; MANNING, E.; COLLINS, M. 2007. Association of fecal shedding of mycobacteria with high ELISA-determined seroprevalence for paratuberculosis in beef herds. JAVMA $230(6): 1-2$

ROUSSEL, A.J.; LIBAL, M.C.; WHITLOCK, R.L.; HAIRGROVE, T.B.; BARLING, K. S.; THOMPSON, J.A. 2005. Prevalence of and risk factors for paratuberculosis in purebred beef cattle. JAVMA. 226 (5):773-778.

SCOTT, H.M.; SORENSEN, O.; WU, J.; CHOW, E.; MANNINEN, K. 2007. Seroprevalence of and agroecological risk factors for Mycobacterium avium subspecies paratuberculosis and Neospora caninum infection among adult beef cattle in cow-calf herds in Alberta, Canada. Can Vet J. 48:397-406.

SILVA, E.; BANDEIRA, D.A. 2005. Diagnóstico da Paratuberculose em Bovinos de Corte do Estado do Pará-Brasil. Dissertação Mestre em Ciência Animal. Universidade Federal do Pará, Empresa Brasileira de Pesquisa Agropecuária, Universidade Federal Rural da Amazônia. Belém,.

SUN, W.; LV, W.; CONG, W.; MENG, Q.; WANG, CH.; SHAN, X.; QIAN, A. 2015. Mycobacterium avium Subspecies paratuberculosis and Bovine Leukemia Virus Seroprevalence and Associated Risk Factors in Commercial Dairy and Beef Cattle in Northern and Northeastern China. BioMed Research International. 2015:ID 315173. 
TUBERQUIA, B.; URIBE, F.; MEDRANO, M.; RAMÍREZ, N; FERNÁNDEZ SILVA, J. 2015 Seroprevalencia de Mycobacterium avium subsp. paratuberculosis y exploración de factores asociados en una población bovina del municipio de Gómez Plata, Antioquia. Rev Colomb Cienc Pecu. 28:104

TURNQUIST, S.; SNIDERIII, T.; KREEGER, J.; MILLER, J.; HAGSTAD, H.; OLCOTT, B. 1991. Serologic evidence of paratuberculosis in Louisiana beef cattle herds as detected by ELISA. Preventive Veterinary Medicine 11 (2):125-130.

VAN HULZEN, K.J.; NIELEN, M.; KOETS, A.P.; DE JONG, G.; VAN ARENDONK, J.A.; HEUVEN, H.C. 2011 Effect of herd prevalence on heritability estimates of antibody response to Mycobacterium avium subspecies paratuberculosis. J Dairy Sci. 2:992-997.

VIR SINGH, S.; DHAMA, K.; CHAUBEY, K.K.; KUMAR, N.; SINGH, P.K.; SOHAL, J.S.; GUPTA, S.; VIR, SINGH-A.; VERMA, A.K.; TIWARI, R.; MAHIMA, CHAKRABORTY, S.; DEB, R. 2013. Impact of host genetics on susceptibility and resistance to Mycobacterium avium subspecies paratuberculosis infection in domestic ruminants. Pak J Biol Sci. 16 (6):251-66.

WALDNER, CH.; CUNNINGHAM, G.; JANZEN, E.; CAMPBELL, J. 2002. Survey of Mycobacterium avium subspecies paratuberculosis serological status in beef herds on community pastures in Saskatchewan. Can Vet J. 43:542-546.

ZAPATA, M.; ARROYAVE, O.; RAMÍREZ, R.; RODAS, J. 2010. MALDONADO, J. Identification of Mycobacterium avium subspecies paratuberculosis by PCR techniques and establishment of control programs for bovine paratuberculosis in dairy herds. Rev Colomb Cienc Pecu. 23:17-27. 